\title{
Lung Ultrasound as a Tool to Evaluate Fluid Accumulation in Dialysis Patients
}

\author{
Maria-Eleni Alexandrou Marieta P. Theodorakopoulou Pantelis A. Sarafidis \\ Department of Nephrology, Hippokration Hospital, Aristotle University of Thessaloniki, Thessaloniki, Greece
}

\section{Keywords}

Hemodialysis · Peritoneal dialysis · Lung ultrasound . Ultrasound B-lines

\begin{abstract}
Background: Volume overload is the main mechanism of BP elevation in end-stage kidney disease (ESKD) patients undergoing hemodialysis or peritoneal dialysis and has been linked to adverse outcomes and increased mortality in this population. Summary: This review discusses current knowledge on lung ultrasound as a tool for detection of extracellular volume overload through evaluation of extravascular lung water content. We describe the principles of lung US, the main protocols to apply it in clinical practice, and accumulated data evidence regarding its associations with cardiovascular events and mortality. We also summarize available evidence on the effect of lung ultrasound-guided volume management strategies on BP control, echocardiographic parameters, and major outcomes in patients undergoing dialysis. Key Messages: Among interventions attempting to reduce the burden of cardiovascular disease in ESKD, effective management of volume overload represents an unmet clinical need. Assessment of hydration status by lung ultrasound is a cheap, easy to employ, and realtime technique that can offer accurate dry weight assessment leading to several clinical benefits.
\end{abstract}

(C) 2022 The Author(s)

Published by S. Karger AG, Basel

\section{Introduction}

Fluid excess is common in ESKD and is one of the main mechanisms associated with high blood pressure (BP) levels, as well as increased cardiac preload and ventricular filling pressures, ultimately leading to left ventricular hypertrophy, heart failure (HF), and death in dialysis patients [1-3]. The cyclic variation in the prevalence of cardiovascular events, demonstrating a day-of-week pattern, is well known in patients undergoing hemodialysis (HD). This is a typical consequence of the intermittent nature of the modality, with interdialytic weight gain (IDWG) alternating with the consequent fast intradialytic volume removal causing intermittent cardiovascular stress [4]. This adverse phenomenon is more pronounced in patients with excessive IDWG [5], or during the long, 3-day interdialytic interval compared to the typical 2-day interdialytic interval $[4,6,7]$. Patients undergoing peritoneal dialysis (PD) are free from this intermittent volume gain and removal; however, the majority of these individuals are also considered to be steadily volume overloaded [8], a fact that also has adverse consequences for the heart structure [9].

The accurate determination of volume status and adopting reliable strategies for the management of fluid accumulation and dry weight adjustment in ESKD patients represent the "holy grail" of the practicing nephrologist for decades [3]. Evaluation of hydration status in the clinical setting has been traditionally guided by clinical karger@karger.com www.karger.com/kbr

Karger $\stackrel{\text { ' }}{5}$
(C) 2022 The Author(s)

Published by S. Karger AG, Basel

This is an Open Access article licensed under the Creative Commons Attribution-NonCommercial-4.0 International License (CC BY-NC) (http://www.karger.com/Services/OpenAccessLicense), applicable to the online version of the article only. Usage and distribution for commercial purposes requires written permission.
Correspondence to:

Pantelis A. Sarafidis, psarafidis11@yahoo.gr 
examination, including assessment of BP, evaluation for presence of lung crackles, pedal edema, or jugular vein distension, and simple diagnostic tools. However, clinical evaluation has been repeatedly shown to have limited diagnostic performance for detecting volume overload and interstitial edema in ESKD and other disease states [10, 11]. In both dialysis modalities, presence of pedal edema did not reliably reflect fluid status compared to parameters obtained from other volume assessment methods and markers $[12,13]$.

Isotope dilution analysis is the gold standard method for body volume assessment; total body water and extracellular volume are preferably determined by deuterium/ tritium dilution and bromide chloride/sucrose dilution, respectively [14]. Combination of dual-energy X-ray absorptiometry with a trace dilution method, in order to overcome limitations from incorrect estimation of lean soft tissue, is superior for determining fat, lean soft, and bone tissue mass composition in dialysis patients [1416]. However, application of these methods in daily clinical routine is unfeasible due to high cost, invasive nature, and poor applicability at bedside. To this end, several diagnostic methods have been developed, including inferior vena cava diameter measurement, echocardiography (i.e., left ventricular [LV] filling pressures and $\mathrm{E} / \mathrm{e}$ ' ratio), biochemical parameters (i.e., BNP/NT-proBNP), use of chest X-ray, and bioimpedance techniques, that are widely used and provide reliable estimates of total tissue fluid content $[3,8,17]$.

In recent years, growing attention has been given to lung ultrasound (US) as a tool to evaluate fluid accumulation in ESKD. This was due to its simple, noninvasive, radiation-free nature and accumulating data on its prognostic utility for cardiovascular events and mortality, as well as its usefulness in accurate dry-weight determination to help BP control and improvement in heart structure [18]. This review discusses the principles of lung US and the current protocols for evaluation of fluid accumulation in dialysis patients, as well as existing evidence regarding its associations with other cardiovascular risk factors and adverse events and its utility in achieving dry weight and improving clinical outcomes.

\section{Principles of Ultrasonography of Pulmonary Parenchyma}

For many decades, lung ultrasonography had been a neglected area, given standard textbook knowledge about the utility of this modality in air-rich organs; that is, the lack of interface of structures with different acoustic impedance in normal lung parenchyma that would permit reflection of the US beam, rendering the lung ultrasound of limited use $[19,20]$. In a normally aerated parenchyma, the beam is scattered, and no structure can be visualized. The only exception to this is the pleural line that produces the characteristic reverberation artifact of a hyperechoic horizontal line (i.e., A-lines) (shown in Fig. 1); this is formed due to multiple reflections of the US beam from the interface of soft tissues of the chest wall and the airfilled alveoli beneath the pleura [21]. The rhythmic respiration-induced movement of this pleural line is known as "lung-sliding," the absence of which constitutes one of the main sonographic criteria of the pneumothorax [22].

During the last decade, this original technical "limitation" of lung US has been transformed to an "advantage": under pathological conditions that decrease the air content and increase the lung density (i.e., presence of extravascular water or blood and consolidation due to an infection or a tumor), partial reflection of the ultrasound beam at deeper zones and creation of other reverberation artifacts or even direct visualization of a solid parenchyma can be enabled [20]. In this context, B-lines, the vertical hyperechoic reverberation artifacts that belong to the family of comet-tail artifacts, represent the reflection of the US beam by thickened subpleural interlobular septa and are considered the sonographic sign of lung interstitial syndrome (Fig. 1) [22]. The image that is formed in this state consists of discrete high-echogenic laser- or comet-tail-like vertical structures that arise from the pleural line and dissipate to the bottom of the screen without fading, moving synchronously with lung sliding [22]. Thus, lung US takes advantage of basic principles of ultrasonography in order to scan for presence and quantify extravascular water excess in the lungs.

Extravascular lung water (EVLW), i.e., the water content of the lung interstitium, constitutes a body fluid compartment of major importance. It is strongly related to pulmonary capillary wedge pressure which reflects the driving force that determines fluid extravasation in the lung, and therefore LV filling pressures, and is the hemodynamic parameter used as the golden standard for optimizing fluid management in the critical care setting [23, 24]. The lung US technique has been previously validated for detection of EVLW against pulmonary artery thermodilution CO measurements after cardiac catheterization. In particular, in a pilot study undertaken in 20 patients evaluated with lung US and the indicator dilution method (PiCCO System) before, immediately after, and $24 \mathrm{~h}$ following cardiac surgery [23], a significant positive correla- 


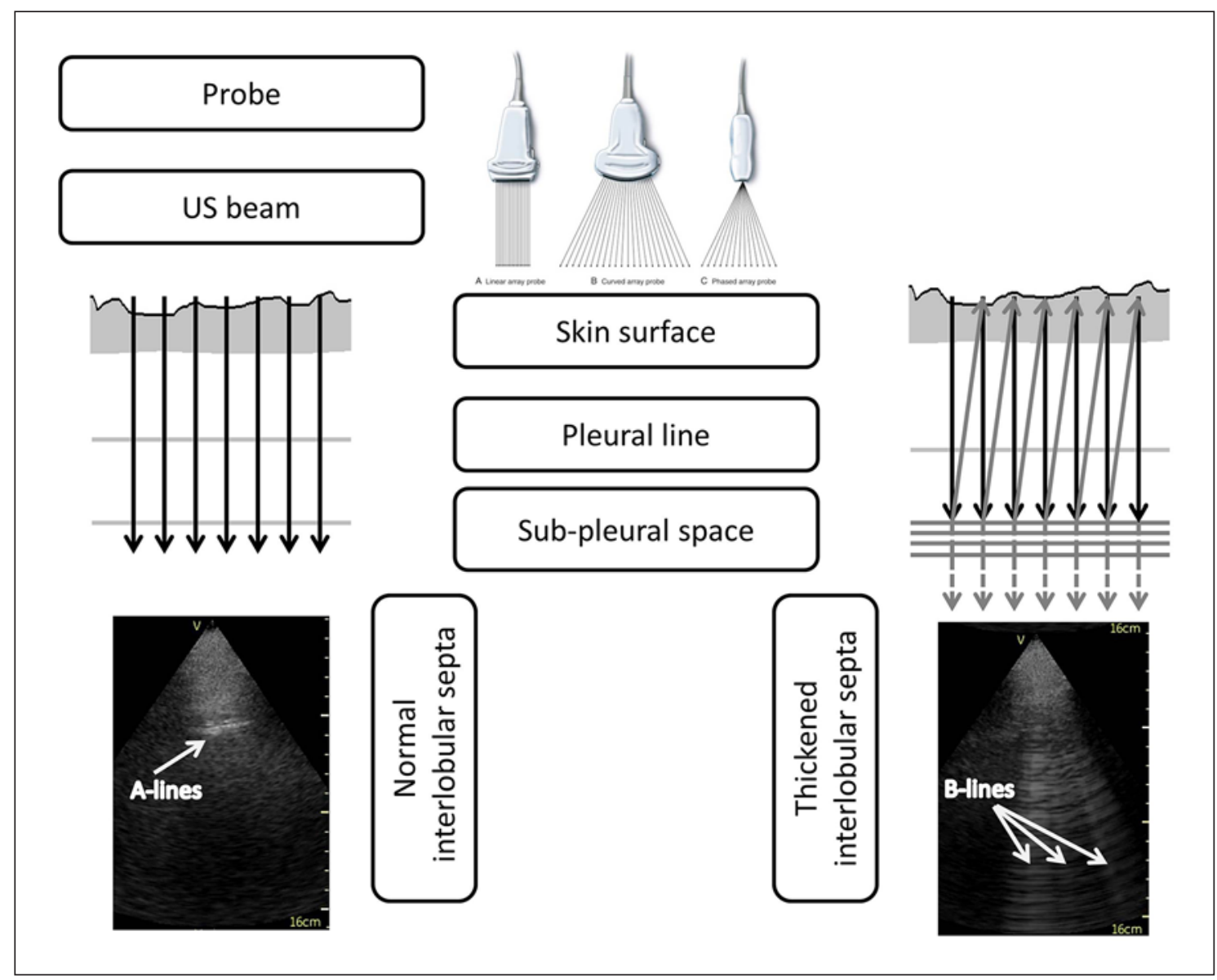

Fig. 1. Principles of lung ultrasound technique and sonographic signs of A- and B-lines in euvolemia state (normal lung water content) on the left and fluid accumulation state (increased lung water content) on the right.

tion was detected between the B-lines score and EVLW determined by PiCCO. In another study with 50 mechanically ventilated patients in the intensive care unit, an even stronger association was observed $(r=0.91, p 0.0001)$ between B-lines score and EVLW by PiCCO. Furthermore, a B-lines score $>1.5$ had a sensitivity and specificity of $92.1 \%$ and $91.7 \%$, respectively, for detection of presence of EVLW above the normal value of $7 \mathrm{~mL} / \mathrm{kg}$ (area under the curve $[\mathrm{AUC}]=0.9419)[25]$.

\section{Lung Scanning Protocols}

The technical requirements to perform a lung US can be easily met. Any conventional 2D scanner may be appropriate, regardless of the presence of Doppler imaging mode capability [20]. Recently, pocket-sized sonographic devices have been developed offering the convenience of portability at a lower expense [26]. Several types of probes have been used, including low-frequency $(2.5-3.5 \mathrm{MHz})$ phased-array, low- to medium-frequency $(3.5-5.0 \mathrm{MHz})$ convex and microconvex, and high-frequency (8.0-12.5 $\mathrm{MHz}$ ) linear probes [27]. The convex and microconvex are the most commonly used types of probe, owing to their intermediate frequency values that allow a reasonable scanning of the pleural line and the subpleural space [20]. The phased-array probe may be also successfully used to detect B-lines that are best visualized with lowfrequency bands and also provide deeper views of the chest, with limited ability in detecting pneumothorax [20]. Whether different types of transducers affect the visualization and therefore quantification of B-lines is still a matter of debate, with one study showing significantly higher scores when using the convex compared to the linear probe [28] and others reporting similar results [23,24, 29]. The penetration depth of the beam should be ideally 


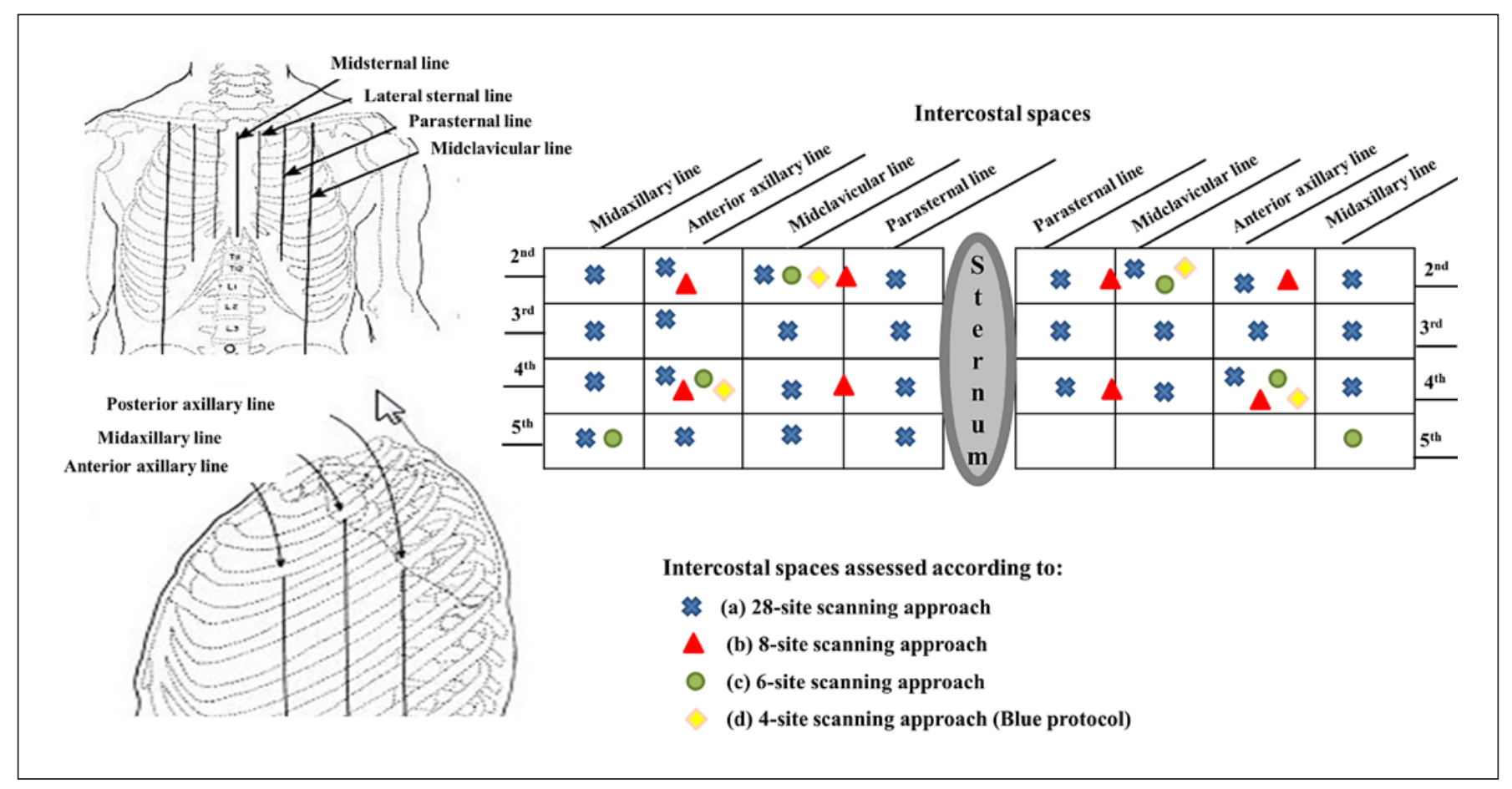

Fig. 2. Graphic depiction of scanned intercostal spaces in two lung ultrasound protocols: the 28-site approach (a), the 8-site approach (b), the 6-site approach (c), and the 4-site approach (d).

set between 4 and $8 \mathrm{~cm}$ from the pleural line, and focus of the image should be set at the level of the pleural line (to achieve maximal concentration of the energy for reflection and reverberation) [27].

Lung US is performed with the probe placed in the intercostal spaces, avoiding the ribs, in an oblique, along the intercostal space, position or in a longitudinal position, perpendicularly to the ribs [20]. In the oblique scanning position, the pleural line is visualized horizontally, covering the major part of the image; in the longitudinal, the pleural line is located in the center and is adjacent to the upper and lower rib that extend to the upper and outer part of the image, forming the so-called bat-sign [30].

During the lung US, the patient may be evaluated in any lying position (supine, prone, or lateral decubitus) or even standing or sitting, since localization of pleural abnormalities, with the exception of pleural effusion, is not immediately altered, and redistribution of EVLW excess tends to remain the same without inducing clinically significant differences [20]. Assessment of lung congestion is traditionally performed with the patient placed firstly in the supine position, with the anterior part of the chest available for scanning, and subsequently in the semi-supine position with the upper body tilted at approximately $45^{\circ}$ to the left decubitus to scan the right axillary lines and subsequently to the right to scan left axillary lines [20]. On the contrary, scanning of the posterior chest is preferred for detection of pulmonary fibrosis. In each intercostal space, the number of visible B-lines is determined either in a quantitative method, by enumerating separate B-lines, or in a semiquantitative method, by estimating the percentage of the image that is occupied by B-lines (i.e., $40 \%$ corresponding to $4 \mathrm{~B}$-lines). In each site, zero defines the complete absence of comet-tail images and thus EVLW content, while ten corresponds to a completely white image, full of comet-tail structures [31]. The total sum of B-lines found in all investigated areas is calculated.

With regard to protocols applied for detection of pulmonary congestion as the source of lung interstitial syndrome, several approaches have been used, with all relevant scanning sites located at the anterior and lateral part of the chest (as shown in Fig. 2). The one most commonly employed is the 28-site zone [31], used in the two aforementioned validation studies of Agricola et al. [23] and Enghard et al. [25], as well as in most observational studies and randomized clinical trials in patients with renal diseases [10, 24, 32-37], including pediatric populations 


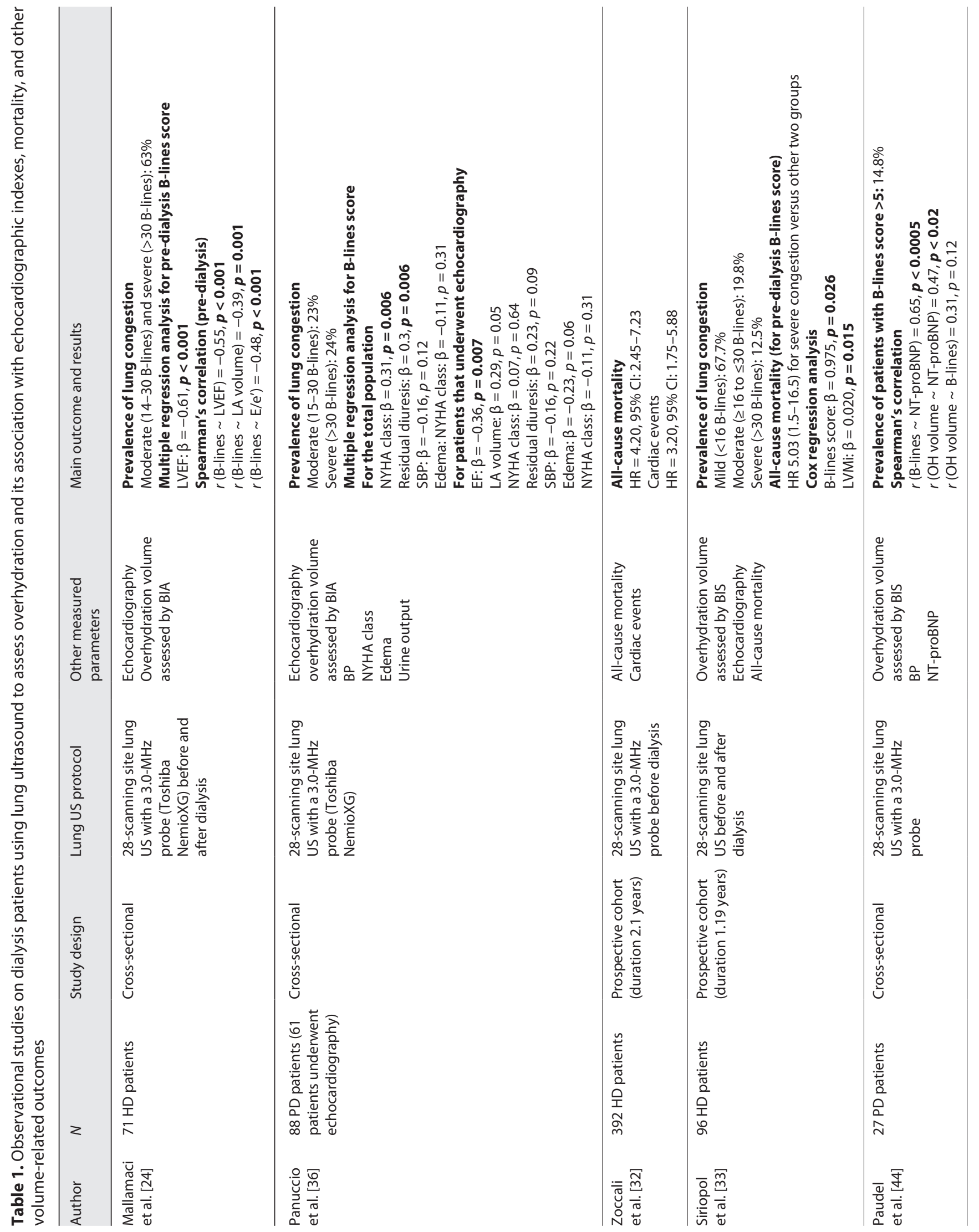




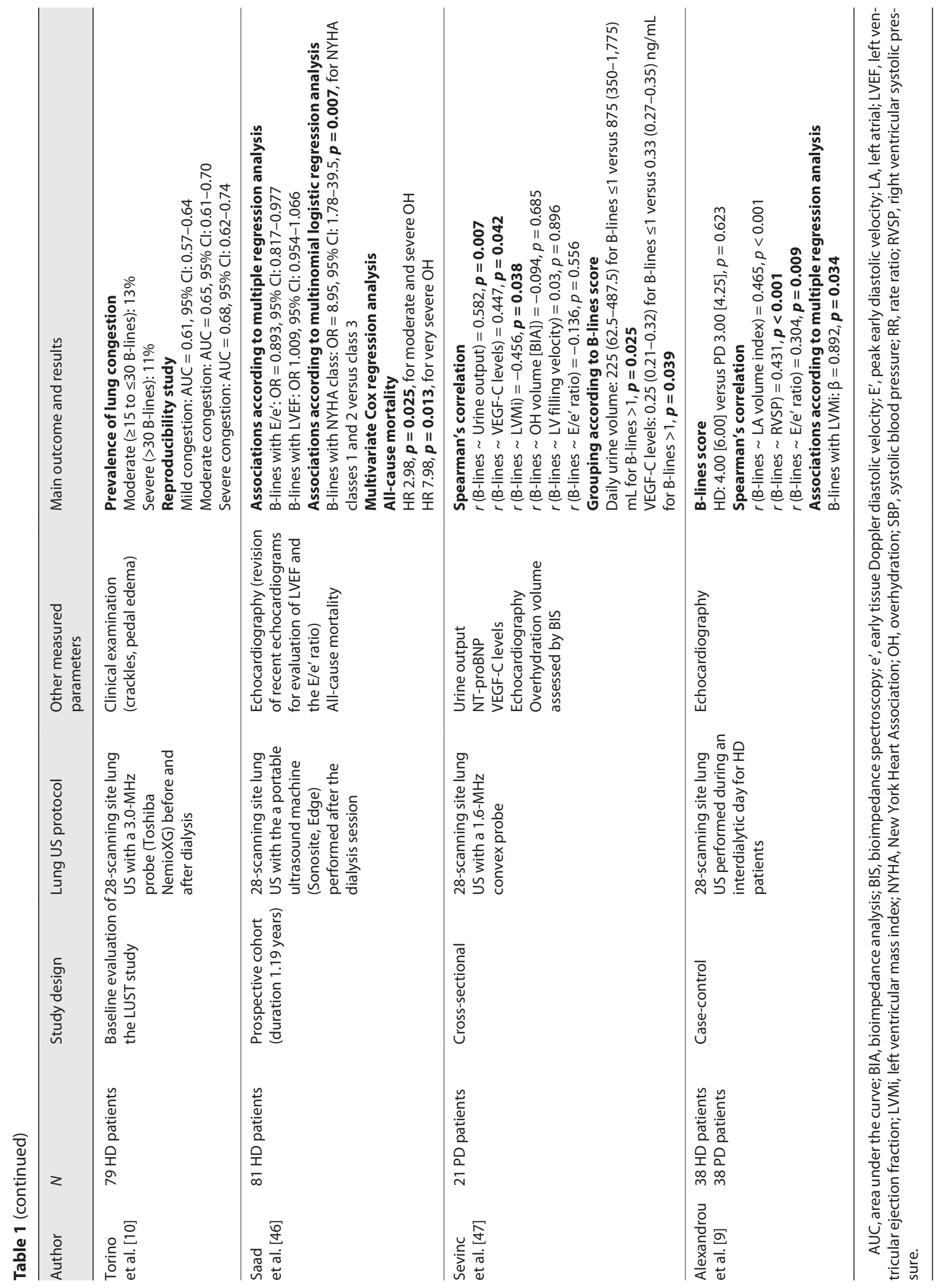


[38]. The study protocol includes scanning from the second to fifth intercostal space on the right and from the second to fourth intercostal space on the left along the parasternal, midclavicular, anterior, and midaxillary lines (16-sites on the right and 12-sites on the left) [31]. According to this approach, a score of $<15$ comets can be characterized as mild pulmonary congestion, a score of $15-30$ comets as moderate, and a score $>30$ comets as severe lung congestion [24].

Other commonly applied protocols, used mainly in the emergency and intensive care settings due to reasons of simplicity, are the 8-point, the 6-point, and the 4-point approaches, shown in Figure 2 [39-42]. The 8-site method includes assessment of two anterior sites (between the sternum and the anterior axillary line) and two lateral sites (between the anterior and posterior axillary line) on each hemithorax. According to this approach that was firstly performed in a study with 300 patients from an emergency unit [39], a score of $>3$ B-lines in a given scanning site is considered positive for detection of lung congestion [42]. In the 6-site approach, that was first implemented in a cohort with 1,005 patients from 7 Italian centers for diagnosis of acute decompensated HF [40], the second space at the midclavicular line, the fourth space at the anterior axillary line, and the fifth space at midaxillary line are scanned at each side, with a positive examination defined as detection of $>3 \mathrm{~B}$-lines in a given or two scanning sites [42]. The 4-site approach, also known as the BLUE protocol, conducted in 260 dyspneic patients in the intensive care unit, is an even more simplified evaluation including scanning of the second intercostal space at the midclavicular line and of the fourth intercostal space at the anterior axillary line, with detection of $>3$ B-lines defining similarly a positive exam [41]. In a study assessing the diagnostic performance of the 4 different scanning site approaches, the 4-site protocol had the lowest C-index $(63.7,95 \%$ CI: 58.5-8.8), an index very similar to AUC, compared to other methods [42]. In ESKD population, the prognostic performance of the 28-site and the 8-site B-lines score for mortality and cardiovascular events was recently studied in a cohort of $303 \mathrm{HD}$ patients, showing a very high interrelation between the two techniques (Spearman's rho $=$ $0.93, p<0.001)$ and a fairly good concordance index $(k=$ 0.79, 95\% CI: 0.74-0.84) [43]. To date, no consensus has been reached regarding the standardization of the procedure or the classification of the overhydration status according to different levels of B-lines total score. However, a properly designed and detailed protocol, executed stepby-step in a repeatable method, is expected to be sufficiently accurate for detecting fluid accumulation.

\section{Observational Studies with Lung Ultrasound in Dialysis Patients}

\section{Prevalence of Volume Overload}

In 2010, Mallamaci et al. [24] undertook the first feasibility study of lung US for detection of fluid accumulation in a population of $71 \mathrm{HD}$ patients, as shown in Table 1 . They found moderate (14-30 comets) and severe (>30 comets) lung congestion in $63 \%$ of patients prior to dialysis session, with the majority of them being fully asymptomatic and persistence of overhydration in $31 \%$ of patients after dialysis. Furthermore, no correlation was shown between B-lines score and bioimpedance-assessed hydration status parameters. In a preliminary analysis of data from baseline evaluation of 79 high cardiovascular risk HD patients in the Lung Water by Ultra-Sound Guided Treatment to Prevent Death and Cardiovascular Complications in High Risk ESRD Patients with Cardiomyopathy Trial (LUST), $13 \%$ of patients were found to be moderately ( $\geq 15$ to $\leq 30$ comets) and $11 \%$ severely ( $>30$ comets) congested [10]. In this study, clinical examination, including crackles and peripheral edema, had a very poor discriminatory capacity to detect any degree of lung water excess evidenced by lung US (AUC for crackles: mild congestion: $\mathrm{AUC}=0.61,95 \% \mathrm{CI}$ : $0.57-0.64$; moderate congestion: $\mathrm{AUC}=0.65,95 \% \mathrm{CI}$ : $0.61-0.70$; severe congestion: $\mathrm{AUC}=0.68,95 \% \mathrm{CI}: 0.62-0.74$ ).

With regard to patients undergoing PD, in a cross-sectional study from Italy with 88 patients, lung US revealed moderate (15-30 B-lines) and severe (>30 B-lines) congestion in $23 \%$ and $24 \%$ of patients, respectively [36]. Both New York Heart Association (NYHA) class and total residual diuresis volume were found to be associated with the B-lines score $\left(\beta_{0}=0.31, p=0.006\right.$, and $\beta_{0}=0.3, p=$ 0.006 , respectively); in contrast, important discordance was noted with results obtained by bioimpedance analysis. In particular, in the subset of 61 patients who underwent echocardiography, only $15 \%$ of those with moderate and $11 \%$ of those with severe lung congestion (classified by lung US) were identified as overhydrated by the bioimpedance technique. In another study with $27 \mathrm{PD}$ patients [44], the prevalence of lung congestion, defined as a B-lines score $>5$, was substantially lower (14.8\%). Moreover, a statistically significant correlation between B-lines score and measured NT-proBNP values was detected $(r=0.65, p<$ $0.0005)$, along with a trend for presence of an association between lung score and volume of overhydration estimated by bioimpedance $(r=0.31, p=0.12)$ [44]. Such results may identify differences in treatment practices regarding volume regulation in different parts of the world. 
Discrepancies in the prevalence of volume overload between lung US and bioimpedance could be attributed to different information on body compartments offered by the two methods. Bioimpedance techniques evaluate fat, lean, and cell mass and provide estimates of total body water (TBW) and extracellular and intracellular water (ECW and ICW, respectively), but cannot discriminate between the two components of the ECW compartment, i.e., the interstitial and intravascular volume. In ESKD patients, loss of lean muscle mass and gain in adipose tissue, as well as hypoalbuminemia, result in increased ECW/ TBW ratio provided by bioimpedance, again without estimates of intravascular fluid compartment [17]. Underestimation of dry weight by bioimpedance was recently reported in malnourished patients with a low fat mass undergoing HD [45].

\section{Associations of Volume Overload Revealed by Lung}

Ultrasound with Echocardiographic Indexes

As shown in Table 1, several observational studies have associated lung water content assessed with lung US with echocardiographic parameters in dialysis patients. The aforementioned study of Mallamaci et al. [24] was also the first to examine this association, showing a significant correlation of B-lines score with $\mathrm{LV}$ ejection fraction (LVEF) before dialysis $(r=-0.55, p<0.001)$, left atrial (LA) volume ( $r=0.39, p=0.001)$, and E/e' $(r=0.48, p$ $<0.001)$, as well as after dialysis. In another study, with 81 HD patients, multiple regression analysis revealed a significant association of B-lines score with E/e', an index of diastolic function (odds ratio $[\mathrm{OR}]=0.893,95 \% \mathrm{CI}$ : 0.817-0.977), but not with LVEF (OR 1.009, 95\% CI: 0.954-1.066) [46].

With regard to PD populations, in the subset of 61 patients who underwent echocardiography in the study of Panuccio et al. [36], the B-lines score was strongly associated with LVEF $(r=-0.40, p=0.002)$ and LA volume $(r$ $=0.30, p=0.020)$; LVEF and LA volume were the only parameters that independently predicted B-lines score in multiple regression analysis $\left(\beta_{0}=-0.36, p=0.007\right.$, and $\beta_{0}$ $=0.29, p=0.05$, respectively) [36]. In a recent small crosssectional study with $21 \mathrm{PD}$ patients, an inverse relationship of B-lines with LV mass index was noted $(r=-0.456$, $p=0.038$ ) [47]. Furthermore, a moderate correlation was observed between B-lines and daily urine output ( $r=$ $0.582, p=0.007)$, suggesting, according to authors, an increase in urine output as a response to overhydration to prevent obvious and symptomatic congestion [47]. In a recently published study of our group, comparing hydration status between $38 \mathrm{HD}$ and $38 \mathrm{PD}$ patients, by means of lung US, no significant differences were noted in the B-lines score between the two dialysis modalities (4.00 [6.00] vs. 3.00 [4.25], $p=0.623$ ). The B-lines score was found to be moderately correlated with LA volume index $(r=0.465, p<0.001)$, right ventricular systolic pressure $(r=0.431, p<0.001)$, and $\mathrm{E} / \mathrm{e}$ ' ratio $(r=0.304, p=0.009)$, but not with LVEF ( $r=-0.166, p=0.153$ ) [9]. According to the results of multiple regression analysis, $\mathrm{B}$-lines score $\left(\beta_{0}=0.892, p=0.034\right)$ and male gender were the only independent predictors of LVMi. All the above results suggest that LV dysfunction and pulmonary congestion may be interlinked conditions, although a causative relationship could not have been established based solely on observational studies.

\section{Associations of Volume Overload Revealed by Lung}

Ultrasound with Cardiovascular Events and Mortality

The association of lung congestion, evaluated by lung US, with mortality and other hard clinical outcomes, which are considered to be related to the harmful effect of chronic volume overload, has been examined so far in 3 observational studies that have been all undertaken in HD populations (Table 1) [32, 33, 46]. In the study of Zoccali et al. [32], including $392 \mathrm{HD}$ patients, the presence of severe lung congestion was associated with a 4.2fold higher risk for death (hazard ratio $[\mathrm{HR}]=4.20,95 \%$ CI: 2.45-7.23) and a 3.2-fold higher risk for cardiac events $(\mathrm{HR}=3.20$, 95\% CI: 1.75-5.88). Siriopol et al. [33] evaluated $96 \mathrm{HD}$ patients and, by comparing parameters obtained by lung US, bioimpedance spectroscopy, and echocardiography, showed that only the pre-dialysis Blines score and LV mass index independently predicted all-cause mortality. Similarly in the study of Saad et al. [46], which studied $81 \mathrm{HD}$ patients after the dialysis session, classification of lung congestion as severe or very severe (>60 lines) was an independent predictor of allcause mortality and cardiac events (HR 2.98 for severe and HR 7.98 for very severe congestion).

\section{Interventional Studies Using Lung Ultrasound for Assessment of Volume Status in Dialysis Patients}

As of this writing, few interventional studies have been undertaken in HD patients aiming to adjust dry weight and optimize volume control and volume-related outcomes using lung US (Table 2). In the Extravascular Lung Water Monitoring by Combined Bioimpedance and Ultrasonography as a Guide for Treatment in Hemodialysis Patients (BUST) study [48], $250 \mathrm{HD}$ patients were ran- 


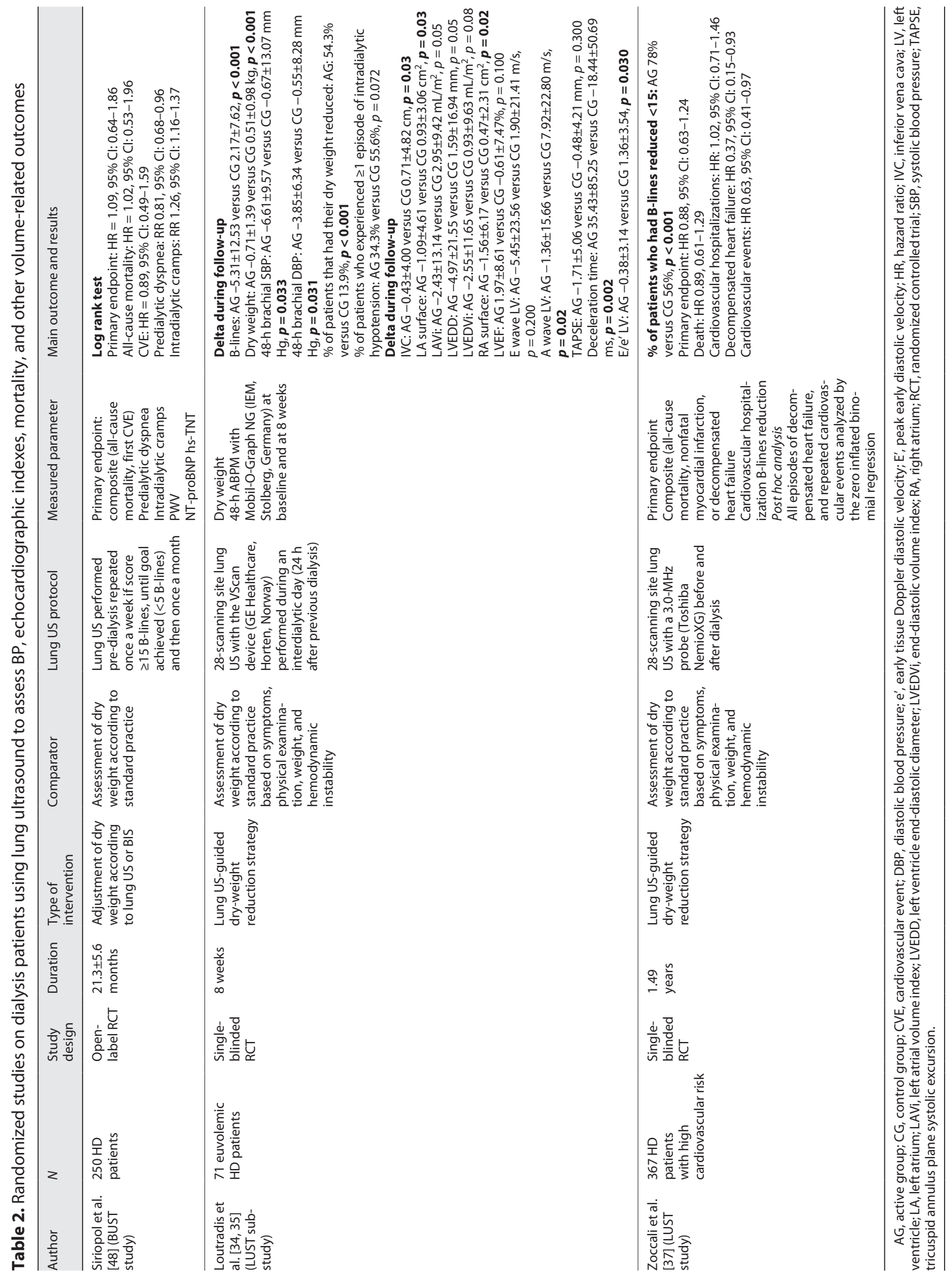




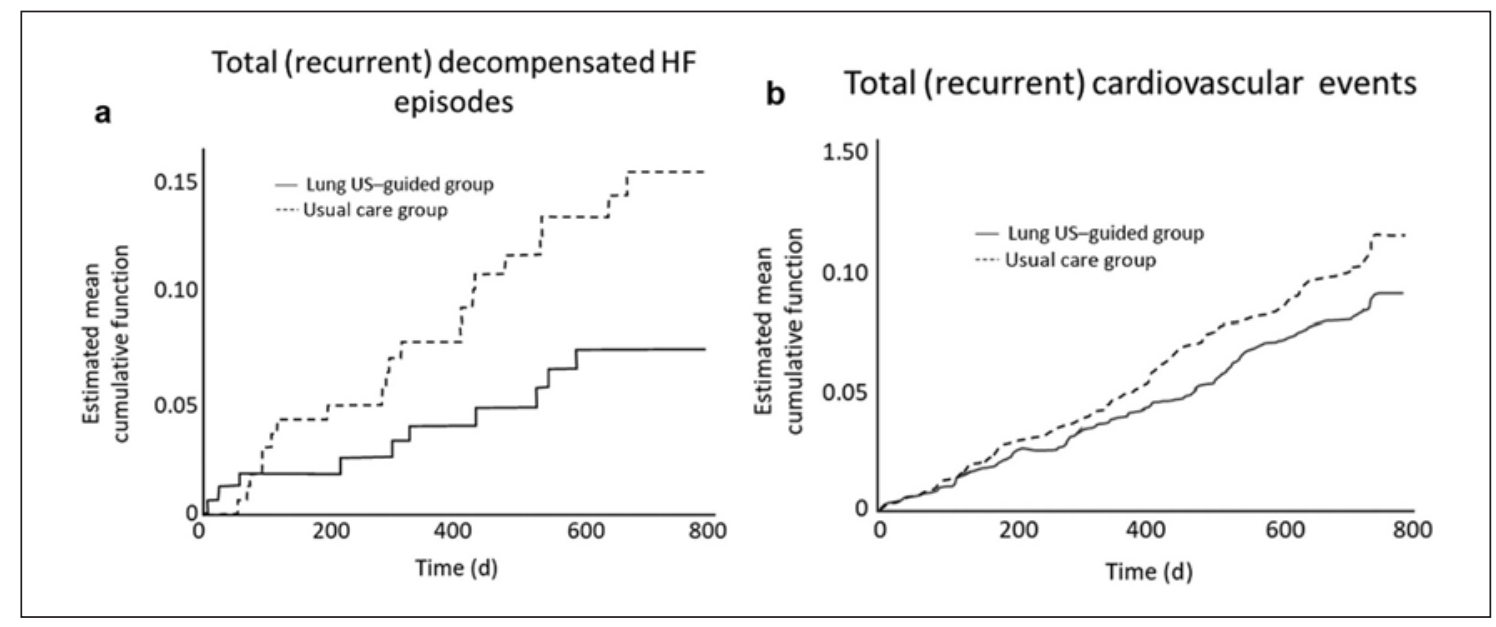

Fig. 3. Cumulative (repeated) episodes of decompensated HF (a) and cardiovascular events (b) in the Lung Water by Ultra-Sound Guided Treatment to Prevent Death and Cardiovascular Complications in High Risk ESRD Patients with Cardiomyopathy Trial (LUST). Reproduced with permission from Zoccali et al. [37].

domized in an active lung US and bioimpedance-guided treatment arm, or in a control group, where dry weight was assessed on clinical criteria. After a median follow-up of 21.3 months, the lung US and bioimpedance-guided strategy was not found to be associated with a reduction in all-cause mortality ( $\mathrm{HR}=1.02,95 \% \mathrm{CI}$ : $0.53-1.96)$ and cardiovascular events (HR $=0.89,95 \%$ CI: 0.49-1.59) compared to standard clinical practice.

The LUST study was an international, multicenter randomized controlled trial where the effect of a lung USguided treatment strategy on major outcomes (all-cause death, nonfatal myocardial infarction, and decompensated HF) was compared to standard-of-care in high-risk HD patients (history of myocardial infarction or unstable angina, acute coronary syndrome, or stable angina pectoris with documented coronary artery disease or HF with NYHA class III-IV) [37]. After a median follow-up of 1.49 years and randomization of 367 patients, no difference between the active and control arm was seen for the primary composite endpoint (all-cause death, nonfatal myocardial infarction, or decompensated HF) (HR 0.88, 95\% CI: $0.63-1.24)$ or the secondary outcome of cardiovascular hospitalization (HR: 1.02, 95\% CI: 0.71-1.46). However, the risk for recurrent decompensated HF episodes (HR 0.37, 95\% CI: 0.15-0.93) and cardiovascular events (HR 0.63 , 95\% CI: 0.41-0.97) was significantly reduced in the active arm (Fig. 3).

A substudy of the LUST trial including 71 hemodialysis patients with hypertension (home $\mathrm{BP} \geq 135 / 85 \mathrm{~mm} \mathrm{Hg}$ covering 6 nondialysis days over a 2 -week period) showed that a lung US-guided treatment strategy for dry-weight probing compared to usual care resulted in a significant reduction of 48-h ambulatory systolic BP after 8 weeks of treatment $(-6.61 \pm 9.57$ vs. $-0.67 \pm 13.07 \mathrm{~mm} \mathrm{Hg}, p=$ 0.033 ) [35]; this BP difference remained after 12 months of follow-up $(-7.78 \pm 13.29$ vs. $-0.10 \pm 14.75 \mathrm{~mm} \mathrm{Hg}, p=$ 0.021 ) [49]. With regard to echocardiographic indexes, a significant reduction in LV filling pressures $\left(E / e^{\prime}-0.38 \pm\right.$ 3.14 vs. $1.36 \pm 3.54, p=0.03$ ) and borderline differences in LA volume $\left(-2.43 \pm 13.14\right.$ vs. $2.95 \pm 9.42 \mathrm{~mL} / \mathrm{m}^{2}, p=$ 0.05 ) were observed in the active group at 8 weeks (Fig. 4) [34]. These were accompanied by a significant reduction in 48 -h PWV $(-0.23 \pm 0.59$ vs. $0.05 \pm 0.45 \mathrm{~m} / \mathrm{s}, p=0.030)$, indicating a positive effect of guided dry-probing on central arterial parameters [50]. In contrast, no differences were shown between the two study groups in $\mathrm{BP}$ variability indexes [51], a fact indirectly supporting that BP lowering should be rather attributed to reduction of volume overload. Of note, intradialytic hypotensive episodes were marginally lower for patients in the active arm of this LUST substudy compared to those that had their dry weight adapted based on standard clinical criteria at 8 weeks ( $34.3 \%$ vs. $55.6 \%, p=0.072)$ [35] and 12 months (71.4\% vs. $88.9 \%, p=0.065)$, despite the fact that more patients in that group had undergone dry-weight reduction $(71.4 \%$ vs. $22.2 \%, p<0.001)$ [49].

The above findings may suggest that detection and quantification of lung congestion via ultrasonography may offer a detailed assessment of fluid overload in a critical interface, enabling strategies based on lung US to objectively assess dry weight and help toward BP reduction and improvement of cardiac chamber dimensions and 


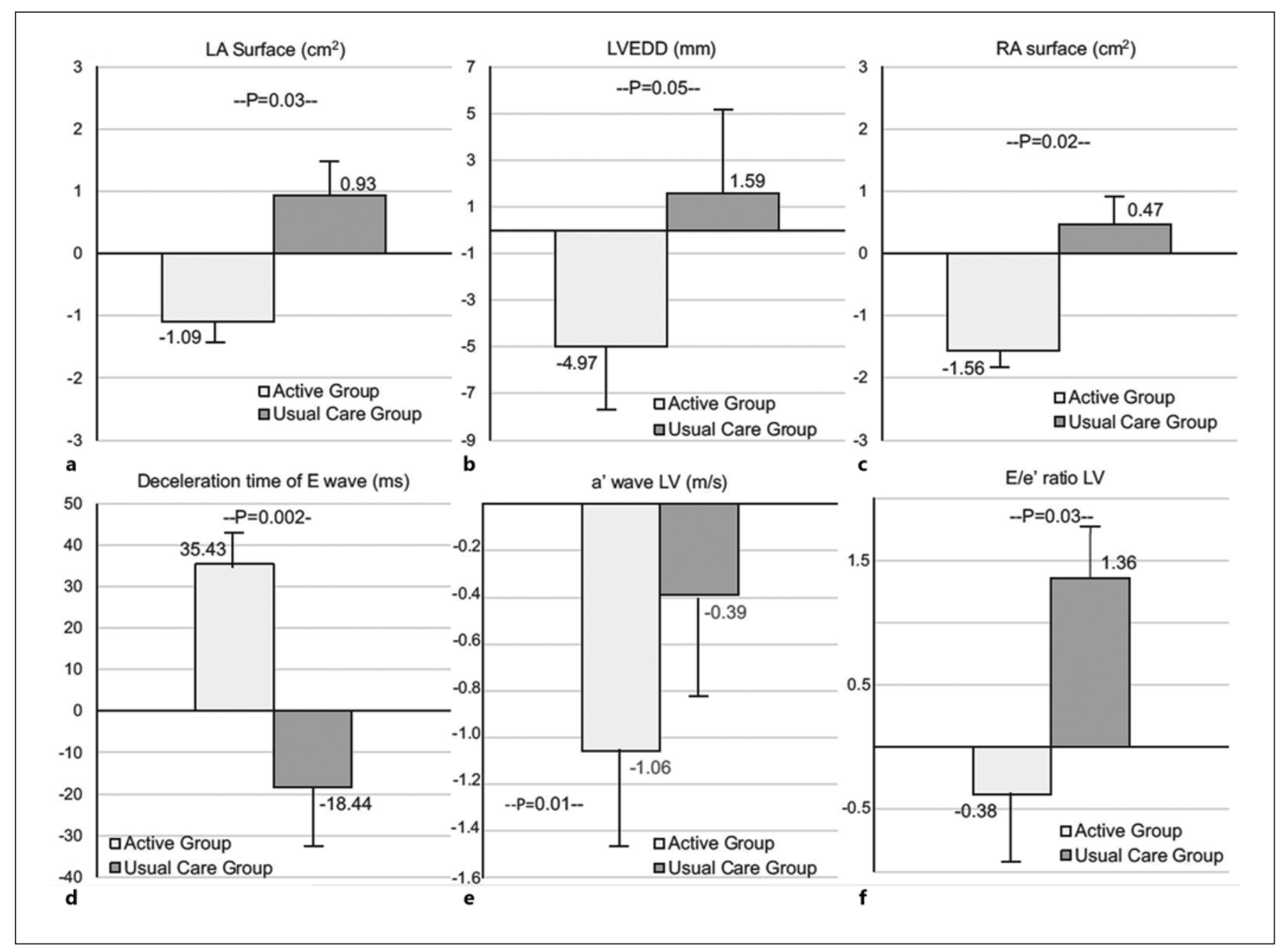

Fig. 4. Between-group comparisons for changes during the study in LA surface (a), LVEDD (b), RA surface (c), E wave deceleration time (d), tissue Doppler peak late a' wave (e), and E/e' LV ratio (f). Reproduced with permission from Loutradis et al. [34]. LA, left atrial; LVEDD, left ventricular end-diastolic diameter; RA, right atrial.

LV diastolic function, without the deleterious effects of intravascular volume depletion. In patients undergoing $\mathrm{PD}$, the effects of US-guided strategy on BP levels, echocardiographic parameters, and cardiovascular outcomes warrant examination by future controlled trials.

\section{Advantages and Limitations of Lung Ultrasound Use in Everyday Clinical Practice for Dialysis Patients}

Lung US is a sonographic technique that possesses all the advantages of a classical US exam: it is an easy to apply, quick (time needed varies between 3 and $10 \mathrm{~min}$ ), noninvasive, noniodized radiation method that may be performed in real time by the patient's bedside $[3,8]$. In the aforementioned feasibility study in HD patients, good interobserver (concordance index $=0.83$, 95\% CI: 0.60 0.93 ) and intraprobe reproducibility was shown, even when assessed with different ultrasound devices [24]. With regard to training demands, the lung US technique is characterized by a steep-learning curve. Results from a web-based training program with 44 participants ( 30 nephrologists and 14 cardiologists) showed a high intraclass correlation coefficient between trainees and the expert trainer $(0.81 \pm 0.21)$ when the first were asked to evaluate 47 videos at the end of the training period. For trainees that had underperformed, the coefficient was adequately increased following additional training [52].

However, this method has some limitations, the most important of which being related to lack of specificity of 
B-lines; these are a sonographic sign of lung interstitial syndrome that cannot discriminate between thickening of interlobular septa due to presence of lung water excess (wet B-lines) and fibrotic thickening (dry B-lines) in pulmonary fibrosis; the latter sign can be found in patients with systemic sclerosis, representing a histological pattern of progressive inflammation and scaring in interstitial lung disease $[27,53]$. Limited capacity to distinguish the etiology of EVLW accumulation (acute respiratory distress syndrome or decompensated HF) has been also reported; however, in the former condition, a more inhomogeneous and irregular pattern may be evident, accompanied by subpleural consolidation, a highly fragmented pleural line, and multiple B-lines alternating with spared areas $[22,30]$. Differentiation of lung water excess from other causes that increase the lung density (i.e., blood and consolidations due to an infection or a tumor) can be easy in most cases based on the patient history and clinical characteristics. However, attention should be given in the interpretation of the exam in patients with evidence or suspicion of such diseases. The cost of the equipment may pose another constraint for lung US application in some low-income environments; however, this seems to be now overridden due to development of newer pocket-size devices that are cheaper. The actual time needed for evaluation of hydration status according to the traditionally used 28-site zone protocol (approximately $8-10 \mathrm{~min}$ ) is not substantially longer than that needed for evaluation by the bioimpedance technique (approximately $5 \mathrm{~min}$ ). If the 8-site zone protocol for lung US is used, then the actual time is substantially shortened [43].

As discussed above, lung US and bioimpedance provide different types of information. Lung US provides estimates of EVLW, i.e., the water content of lung interstitium, a body compartment where volume excess can have direct consequences. Bioimpedance provides information on TBW, ECW, and ICW excess. As such, the two methods should be considered complementary and not competitive. In settings where the cost of lung US is an issue, evaluation with this procedure should be preferred over bioimpedance for patients in whom it is critical to know what is the situation in the lung interface, such as patients with borderline cardiac function and repeated episodes of congestive heart failure, or patients with increased ECW according to bioimpedance due to hypoalbuminemia or poor nutrition status, as bioimpedance cannot discriminate between increase in interstitial fluid and intravascular volume.

With regard to the use of lung US over natriuretic peptides, such as BNP, NT-proBNP, and most recently MR-
proANP, while the latter are considered valid markers of fluid accumulation in patients with heart failure, they cannot substitute lung US in patients with ESKD. Both BNP and NT-proBNP are passively filtered by the glomerulus, and while BNP is degraded by neprilisyn at the proximal convoluted tubule, NT-proBNP (having no neprylisin binding domain) is not degraded by neprylisin and has therefore a slower clearance. Therefore, their levels are increased in the context of reduced GFR, and presence of $\mathrm{CKD}$ is considered one of the main caveats in interpretation of their measurements [54,55]. In ESKD, this interpretation is even more complex, as the majority of dialysis patients have no considerable residual renal function, while removal through hemodialysis session may also interfere with their serum levels [56]. Further, in ESKD, their levels are strongly affected by cardiac systolic dysfunction [54]. As such, lung US is considered a more valid method for volume overload estimation in dialysis than natriuretic peptides.

\section{Conclusion}

Lung ultrasound is a cheap, noninvasive, nonionized radiation, portable, real-time method to evaluate volume overload in ESKD patients that has gained growing attention in the last decade. It provides reliable estimates of EVLW content as shown in validation studies against thermodilution measurements of wedge pressures after cardiac catheterization. It is a method with sufficiently good interobserver and intraprobe reproducibility when performed by trained physicians. In patients undergoing $\mathrm{HD}$, a strong correlation between ultrafiltration volume and decrease in B-lines sum, and therefore interstitial lung congestion, has been observed during dialysis session. Furthermore, a strong correlation of B-lines score and LV mass and indices of LV systolic and diastolic performance has been shown, as well as a predictive value for cardiovascular morbidity and mortality. Results from randomized controlled trials show a beneficial effect of US-guided dry weight probing on ambulatory BP levels, central pressures, arterial stiffness, and echocardiographic indexes, as well as a risk reduction of recurrent episodes of decompensated HF compared to standard practice based on clinical assessment. These were accompanied by reduced episodes of intradialytic hypotension which could lead to decreased end-organ perfusion. For all the above reasons, lung US can be a quite useful tool for the practicing nephrologist in the complex quest for volume management and should rather be integrated in training programs and future recommendations. 


\section{Conflict of Interest Statement}

P. Sarafidis has received research support for the LUST ABPM substudy by ERA-EDTA. The other authors have no conflicts of interest to declare.

\section{Funding Sources}

The authors certify that there are no sources of financial grants or other funding that are relevant to this paper and that should be acknowledged.

\section{References}

1 Dekker MJE, Marcelli D, Canaud BJ, Carioni P, Wang Y, Grassmann A, et al. Impact of fluid status and inflammation and their interaction on survival: a study in an international hemodialysis patient cohort. Kidney Int. 2017;91(5):1214-23.

2 Konings CJ, Kooman JP, Schonck M, Dammers R, Cheriex E, Palmans Meulemans AP, et al. Fluid status, blood pressure, and cardiovascular abnormalities in patients on peritoneal dialysis. Perit Dial Int. 2002 Aug;22(4):477-87.

3 Loutradis C, Sarafidis PA, Ferro CJ, Zoccali C. Volume overload in hemodialysis: diagnosis, cardiovascular consequences, and management. Nephrol Dial Transplant. 2021 Dec; 36(12):2182-93.

4 Loutradis C, Sarafidis PA, Papadopoulos CE, Papagianni A, Zoccali C. The ebb and flow of echocardiographic cardiac function parameters in relationship to hemodialysis treatment in patients with ESRD. J Am Soc Nephrol. 2018 May;29(5):1372-81.

5 Kalantar-Zadeh K, Regidor DL, Kovesdy CP, Van Wyck D, Bunnapradist S, Horwich TB, et al. Fluid retention is associated with cardiovascular mortality in patients undergoing long-term hemodialysis. Circulation. 2009 Feb;119(5):671-9.

6 Koutroumbas G, Georgianos PI, Sarafidis PA, Protogerou A, Karpetas A, Vakianis P, et al. Ambulatory aortic blood pressure, wave reflections and pulse wave velocity are elevated during the third in comparison to the second interdialytic day of the long interval in chronic haemodialysis patients. Nephrol Dial Transplant. 2015 Apr;30(12):2046-53.

7 Tsilonis K, Sarafidis PA, Kamperidis V, Loutradis C, Georgianos PI, Imprialos K, et al. Echocardiographic parameters during long and short interdialytic intervals in hemodialysis patients. Am J Kidney Dis. 2016 Nov; 68(5):772-81.

8 Alexandrou ME, Balafa O, Sarafidis P. Assessment of hydration status in peritoneal dialysis patients: validity, prognostic value, strengths, and limitations of available techniques. Am J Nephrol. 2020;51(8):589-612.

9 Alexandrou ME, Sarafidis PS, Theodorakopoulou MP, Sachpekidis V, Papadopoulos C,

\section{Author Contributions}

Review concept and design: P.S. Literature search in databases: M.-E.A. and M.T. Drafting of the manuscript: M.-E.A. Critical revision of the manuscript for important intellectual content: P.S. All authors contributed important intellectual content during manuscript drafting and have read and approved the final version of the manuscript.
Loutradis C, et al. Cardiac geometry, function, and remodeling patterns in patients under maintenance hemodialysis and peritoneal dialysis treatment. Ther Apher Dial. 2021. Epub ahead of print.

10 Torino C, Gargani L, Sicari R, Letachowicz K, Ekart R, Fliser D, et al. The agreement between auscultation and lung ultrasound in hemodialysis patients: the LUST study. Clin J Am Soc Nephrol. 2016 Nov; 11(11):2005-11.

11 Damy T, Kallvikbacka-Bennett A, Zhang J, Goode K, Buga L, Hobkirk J, et al. Does the physical examination still have a role in patients with suspected heart failure? Eur J Heart Fail. 2011 Dec;13(12):1340-8.

12 Agarwal R, Andersen MJ, Pratt JH. On the importance of pedal edema in hemodialysis patients. Clin J Am Soc Nephrol. 2008 Jan;3(1): 153-8.

13 Ferreira-Filho SR, Machado GR, Ferreira VC, Rodrigues CFMA, Proença de Moraes T, Divino-Filho JC, et al. Back to basics: pitting edema and the optimization of hypertension treatment in incident peritoneal dialysis patients (BRAZPD). PLoS One. 2012;7(5): e36758.

14 Woodrow G, Oldroyd B, Turney JH, Davies PS, Day JM, Smith MA. Four-component model of body composition in chronic renal failure comprising dual-energy X-ray absorptiometry and measurement of total body water by deuterium oxide dilution. Clin Sci. 1996 Dec;91(6):763-9.

15 Lee SY, Gallagher D. Assessment methods in human body composition. Curr Opin Clin Nutr Metab Care. 2008 Sep;11(5):566-72.

16 Locatelli F, Fouque D, Heimburger O, Drüeke TB, Cannata-Andía JB, Hörl WH, et al. Nutritional status in dialysis patients: a European consensus. Nephrol Dial Transplant. 2002 Apr;17(4):563-72.

17 Davies SJ, Davenport A. The role of bioimpedance and biomarkers in helping to aid clinical decision-making of volume assessments in dialysis patients. Kidney Int. 2014 Sep;86(3):489-96.

18 Flythe JE, Chang TI, Gallagher MP, Lindley E, Madero M, Sarafidis PA, et al. Blood pressure and volume management in dialysis: conclu- sions from a Kidney Disease: Improving Global Outcomes (KDIGO) Controversies Conference. Kidney Int. 2020 May;97(5): 861-76.

19 Gargani L. Lung ultrasound: a new tool for the cardiologist. Cardiovasc Ultrasound. 2011 Feb;9:6.

20 Gargani L, Volpicelli G. How i do it: lung ultrasound. Cardiovasc Ultrasound. 2014 Jul; 12:25.

21 Volpicelli G. Lung sonography. J Ultrasound Med. 2013 Jan;32(1):165-71.

22 Volpicelli G, Elbarbary M, Blaivas M, Lichtenstein DA, Mathis G, Kirkpatrick AW, et al. International evidence-based recommendations for point-of-care lung ultrasound. Intensive Care Med. 2012 Apr;38(4): 577-91.

23 Agricola E, Bove T, Oppizzi M, Marino G, Zangrillo A, Margonato A, et al. "Ultrasound comet-tail images": a marker of pulmonary edema: a comparative study with wedge pressure and extravascular lung water. Chest. 2005 May; 127(5):1690-5.

24 Mallamaci F, Benedetto FA, Tripepi R, Rastelli S, Castellino P, Tripepi G, et al. Detection of pulmonary congestion by chest ultrasound in dialysis patients. JACC Cardiovasc Imaging. 2010 Jun;3(6):586-94.

25 Enghard P, Rademacher S, Nee J, Hasper D, Engert U, Jörres A, et al. Simplified lung ultrasound protocol shows excellent prediction of extravascular lung water in ventilated intensive care patients. Crit Care. 2015 Feb;19:36.

26 Sicari R, Galderisi M, Voigt J-U, Habib G, Zamorano JL, Lancellotti P, et al. The use of pocket-size imaging devices: a position statement of the European Association of Echocardiography. Eur J Echocardiogr. $2011 \mathrm{Feb}$; 12(2):85-7.

27 Covic A, Siriopol D, Voroneanu L. Use of lung ultrasound for the assessment of volume status in CKD. Am J Kidney Dis. 2018 Mar; 71(3):412-22

28 Sperandeo M, Varriale A, Sperandeo G, Polverino E, Feragalli $B$, Piattelli ML, et al. Assessment of ultrasound acoustic artifacts in patients with acute dyspnea: a multicenter study. Acta Radiol. 2012 Oct;53(8):885-92. 
29 Reissig A, Kroegel C. Transthoracic sonography of diffuse parenchymal lung disease: the role of comet tail artifacts. J Ultrasound Med. 2003 Feb;22(2):173-80.

30 Lichtenstein DA. Lung ultrasound in the critically ill. Ann Intensive Care. 2014 Jan;4(1):1.

31 Jambrik Z, Monti S, Coppola V, Agricola E, Mottola G, Miniati M, et al. Usefulness of ultrasound lung comets as a nonradiologic sign of extravascular lung water. Am J Cardiol. 2004 May;93(10):1265-70.

32 Zoccali C, Torino C, Tripepi R, Tripepi G, D’Arrigo G, Postorino M, et al. Pulmonary congestion predicts cardiac events and mortality in ESRD. J Am Soc Nephrol. 2013 Mar; 24(4):639-46.

33 Siriopol D, Hogas S, Voroneanu L, Onofriescu M, Apetrii M, Oleniuc M, et al. Predicting mortality in haemodialysis patients: a comparison between lung ultrasonography, bioimpedance data and echocardiography parameters. Nephrol Dial Transplant. 2013 Nov:28(11):2851-9.

34 Loutradis C, Papadopoulos CE, Sachpekidis V, Ekart R, Krunic B, Karpetas A, et al. Lung ultrasound-guided dry weight assessment and echocardiographic measures in hypertensive hemodialysis patients: a randomized controlled study. Am J Kidney Dis. 2020; 75(1):11-20.

35 Loutradis C, Sarafidis PA, Ekart R, Papadopoulos C, Sachpekidis V, Alexandrou ME, et al. The effect of dry-weight reduction guided by lung ultrasound on ambulatory blood pressure in hemodialysis patients: a randomized controlled trial. Kidney Int. 2019 Jun; 95(6):1505-13.

36 Panuccio V, Enia G, Tripepi R, Torino C, Garozzo M, Battaglia GG, et al. Chest ultrasound and hidden lung congestion in peritoneal dialysis patients. Nephrol Dial Transplant. 2012 Sep;27(9):3601-5.

37 Zoccali C, Torino C, Mallamaci F, Sarafidis P, Papagianni A, Ekart R, et al. A randomized multicenter trial on a lung ultrasound-guided treatment strategy in patients on chronic hemodialysis with high cardiovascular risk. Kidney Int. 2021 Dec;100(6):1325-33.

$38 \mathrm{Fu} \mathrm{Q}$, Chen Z, Fan J, Ling C, Wang X, Liu X, et al. Lung ultrasound methods for assessing fluid volume change and monitoring dry weight in pediatric hemodialysis patients. $\mathrm{Pe}-$ diatr Nephrol. 2021 Apr;36(4):969-76.
39 Volpicelli G, Mussa A, Garofalo G, Cardinale L, Casoli G, Perotto F, et al. Bedside lung ultrasound in the assessment of alveolar-interstitial syndrome. Am J Emerg Med. 2006 Oct; 24(6):689-96.

40 Pivetta E, Goffi A, Lupia E, Tizzani M, Porrino G, Ferreri E, et al. Lung ultrasound-implemented diagnosis of acute decompensated heart failure in the ED: a SIMEU multicenter study. Chest. 2015 Jul;148(1):202-10.

41 Lichtenstein DA, Mezière GA. Relevance of lung ultrasound in the diagnosis of acute respiratory failure: the BLUE protocol. Chest. 2008 Jul;134(1):117-25.

42 Buessler A, Chouihed T, Duarte K, Bassand A, Huot-Marchand M, Gottwalles Y, et al. Accuracy of several lung ultrasound methods for the diagnosis of acute heart failure in the ED: a multicenter prospective study. Chest. 2020 Jan;157(1):99-110.

43 Torino C, Tripepi R, Loutradis C, Sarafidis P, Tripepi G, Mallamaci F, et al. Can the assessment of ultrasound lung water in haemodialysis patients be simplified? Nephrol Dial Transplant. 2021 Dec 2;36(12):2321-6.

44 Paudel K, Kausik T, Visser A, Ramballi C, Fan SL. Comparing lung ultrasound with bioimpedance spectroscopy for evaluating hydration in peritoneal dialysis patients. Nephrology. 2015 Jan;20(1):1-5.

45 Kim H-R, Jeon J-W, Bae H-J, Shin J-A, Ham $\mathrm{Y}-\mathrm{R}, \mathrm{Na} \mathrm{K}-\mathrm{R}$, et al. Body fat plays an important role in of bioimpedance spectroscopybased dry weight measurement error for patients with hemodialysis. Diagnostics. 2021 Oct;11(10):1907.

46 Saad MM, Kamal J, Moussaly E, Karam B, Mansour W, Gobran E, et al. Relevance of Blines on lung ultrasound in volume overload and pulmonary congestion: clinical correlations and outcomes in patients on hemodialysis. Cardiorenal Med. 2018;8(2):83-91.

47 Sevinc M, Hasbal NB, Basturk T, Ozcafer PN, Kocas BB, Kilickesmez K, et al. Comparison of lung ultrasound and other volumetric methods in peritoneal dialysis patients. Medicine. 2021 Jan;100(3):e23856.
48 Siriopol D, Onofriescu M, Voroneanu L, Apetrii M, Nistor I, Hogas S, et al. Dry weight assessment by combined ultrasound and bioimpedance monitoring in low cardiovascular risk hemodialysis patients: a randomized controlled trial. Int Urol Nephrol. 2017 Jan;49(1): 143-53.

49 Loutradis C, Sarafidis PA, Ekart R, Tsouchnikas I, Papadopoulos C, Kamperidis V, et al. Ambulatory blood pressure changes with lung ultrasound-guided dry-weight reduction in hypertensive hemodialysis patients: 12-month results of a randomized controlled trial. J Hypertens. 2021 Jul;39(7):1444-52.

50 Loutradis C, Papagianni A, Ekart R, Theodorakopoulou M, Minopoulou I, Pagourelias E, et al. Excess volume removal following lung ultrasound evaluation decreases central blood pressure and pulse wave velocity in hemodialysis patients: a LUST sub-study. J Nephrol. 2020 Dec;33(6):1289-300.

51 Loutradis C, Sarafidis PA, Theodorakopoulou M, Ekart R, Alexandrou ME, Pyrgidis N, et al. Lung ultrasound-guided dry-weight reduction in hemodialysis patients does not affect short-term blood pressure variability. Am J Hypertens. 2019 Jul;32(8):786-95.

52 Gargani L, Sicari R, Raciti M, Serasini L, Passera M, Torino C, et al. Efficacy of a remote web-based lung ultrasound training for nephrologists and cardiologists: a LUST trial sub-project. Nephrol Dial Transplant. 2016 Dec;31(12):1982-8.

53 Picano E, Pellikka PA. Ultrasound of extravascular lung water: a new standard for pulmonary congestion. Eur Heart J. 2016 Jul; 37(27):2097-104.

54 Wang AY, Lai KN. Use of cardiac biomarkers in end-stage renal disease. J Am Soc Nephrol. 2008 Sep;19(9):1643-52.

55 Gaggin HK, Januzzi JL. The past, the present, and the future of natriuretic peptides in the diagnosis of heart failure. Eur Heart J Suppl. 2018 Aug;20(Suppl G):G11-20.

56 Safley DM, Awad A, Sullivan RA, Sandberg KR, Mourad I, Boulware M, et al. Changes in B-type natriuretic peptide levels in hemodialysis and the effect of depressed left ventricular function. Adv Chronic Kidney Dis. 2005 Jan;12(1):117-24. 